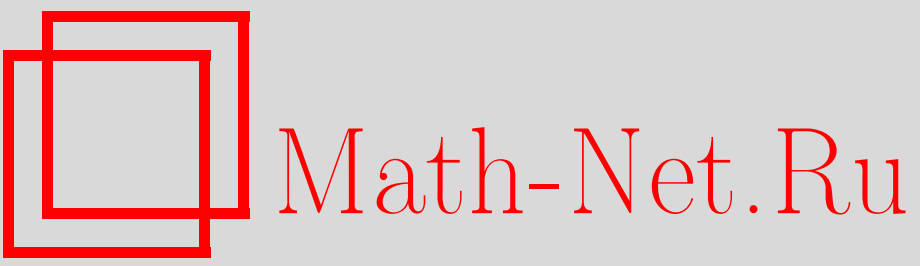

И. К. Бабенко, Сильная интерсистолическая мягкость замкнутых многообразий, УМH, 2000, том 55, выпуск 5, 171-172

DOI: https://doi.org/10.4213/rm324

Использование Общероссийского математического портала Math-Net.Ru подразумевает, что вы прочитали и согласны с пользовательским соглашением

http://www.mathnet.ru/rus/agreement

Параметры загрузки:

IP: 54.174 .149 .18

26 апреля 2023 г., $14: 11: 18$ 


\title{
СИЛЬНАЯ ИНТЕРСИСТОЛИЧЕСКАЯ МЯГКОСТЬ ЗАМКНУТЫХ МНОГООБРАЗИЙ
}

\author{
И. К. БАБЕНКО
}

Пусть $(M, g)$ - риманово многообразие размерности $m$. Если $\Delta^{p}$ - стандартный $p$-мерный симплекс, то для каждого сингулярного липшицева $p$-мерного симплекса $\tau^{p}: \Delta^{p} \rightarrow M$ естественным образом определен его объем $\operatorname{vol}_{p}(\tau, g)$ по отношению к индуцированной метрике, которая определена почти всюду и измерима. Поле коэффициентов $\mathbf{k}$ будет в дальнейшем либо $\mathbb{Z}$, либо $\mathbb{R}$. Для липшицевой сингулярной $p$-цепи

$$
\alpha(p)=\sum_{i} r_{i} \tau_{i}^{p}, \quad r_{i} \in \mathbf{k},
$$

определим ее объем, положив $\operatorname{vol}_{p}(\alpha(p), g)=\sum_{i}\left|r_{i}\right| \operatorname{vol}_{p}\left(\tau_{i}^{p}, g\right)$. Для каждого целочисленного класса гомологий $a \in \mathbb{H}_{p}(M, \mathbb{Z})$ зададим его объем равенством:

$$
\operatorname{vol}_{p}(\alpha, g)=\inf _{\alpha(\mathbb{Z}) \in a} \operatorname{vol}_{p}(\alpha(\mathbb{Z}), g),
$$

где нижняя грань берется по всем целочисленным сингулярным липшицевым цепям $\alpha(\mathbb{Z})$, представляющим класс $a$.

Хорошо известно [1], что усреднение (1) приводит к "массе" соответствуюшего класса гомологий (определения и свойства массы и комассы можно найти в [2]):

$$
\operatorname{mass}(a, g)=\lim _{l \rightarrow \infty} \frac{1}{l} \operatorname{vol}_{p}(l a, g) .
$$

Теперь определим $p$-систолу по модулю кручения для риманова многообразия $(M, g)$, положив:

$$
\operatorname{sys}_{p}(M, g)=\inf _{\substack{a \in \mathbb{H}_{p}(M, \mathbb{Z}) \\ a \neq 0 \text { mod Tors }}} \operatorname{vol}_{p}(a, g) .
$$

Соответственно стабильную $p$-систолу многообразия $(M, g)$ определим как

$$
\operatorname{sys}_{\operatorname{stab}}(M, g)=\inf _{\substack{a \in \mathbb{H}_{p}(M, \mathbb{Z}) \\ a \neq 0 \text { mod Tors }}} \operatorname{mass}(a, g) \text {. }
$$

Для любого многообразия $(M, g)$ имеет место очевидное неравенство:

$$
\operatorname{sys}_{p}(M, g) \geqslant \operatorname{sys} \operatorname{stab}_{p}(M, g),
$$

причем простые примеры показывают, что две эти величины могут достаточно сильно различаться.

Сравнительно давно известен ряд систолических и интерсистолических проблем, с которыми можно познакомиться по прекрасной лекции [3]. Суть этих проблем кратко можно сформулировать следующим образом: для данного замкнутого многообразия $M$ можно ли, меняя метрику в классе метрик единичного объема, сделать $p$-систолу или произведение систол разных (например, дополнительных) размерностей сколь угодно большим числом?

Интенсивное развитие систолической геометрии в последнее десятилетие показывает, что для систол размерности больше единицы ответ на этот вопрос очень часто бывает положительным, см. [4]-[9].

В частности, в [6] при дополнительных топологических предположениях на многообразии $M$ установлена его $(p, m-p)$-систолическая мягкость. В действительности эти дополнительные условия являются излишними, и мягкость имеет место для любого замкнутого многообразия. Перейдем к точным определениям и формулировкам. 
ОПРЕДЕЛЕНИЕ. Замкнутое $m$-мерное многообразие $M$ называется $(p, m-p)$-мягким $(1 \leqslant p \leqslant m / 2)$, если

$$
\inf _{g} \frac{\operatorname{vol}_{m}(M, g)}{\operatorname{sys}_{p}(M, g) \cdot \operatorname{sys}_{m-p}(M, g)}=0
$$

где нижняя грань берется по всем римановым метрикам $g$ на $M$.

Основным результатом этой заметки является

ТЕОРемА. Для любого замкнутого $(1 \leqslant p<m / 2)$ имеет место равенство:

$$
\inf _{g} \frac{\operatorname{vol}(M, g)}{\operatorname{sys}_{p}(M, g) \cdot \operatorname{sys~stab}_{m-p}(M, g)}=0 .
$$

СлеДСтвиЕ. Любое замкнутое $m$-мерное многообразие $M$ является $(p, m-p)$-мягким для всех $1 \leqslant p \leqslant m / 2$.

ЗАмечАния. 1. Для $p<m / 2$ это непосредственно вытекает из теоремы с учетом (3). При $m=2 p$ это доказано в [7].

2. Хорошо известно, что заменить $\operatorname{sys}_{p}$ на $\operatorname{sys} \operatorname{stab}_{p}$ в (5) нельзя, см., например, [10]. Автору не известно, что будет происходить при вариации метрики с дробью (5), если $m=2 p$.

Доказательство теоремы основано на изучении систолической геометрии на симплициальных комплексах специального вида. Каждый конечный симплициальный комплекс обладает римановыми метриками, определенными и гладкими на каждой грани и с естественными условиями согласованности на пересекающихся симплексах. Кроме того, на каждом симплициальном комплексе определен комплекс внешних дифференциальных форм, впервые введенный Томом еше в пятидесятые годы.

Все это превращает симплициальные комплексы в объекты, близкие к римановым многообразиям, но в отличие от многообразий класс конечных симплициальных комплексов замкнут относительно операции приклеивания по симплициальному отображению. Подробное доказательство будет опубликовано.

\section{СПИСОК ЛИТЕРАТУРЫ}

[1] Federer H. // Indiana Univ. Math. J. 1974. V. 24. P. 351-407. [2] Федерер Г. Геометрическая теория меры. М.: Наука, 1987. [3] Berger М. // Astérisque. 1993. V. 216. P. 279-310. [4] Katz M. // Geom. Dedicata. 1995. V.57. P. 195-206. [5] Berard-Bergery L., Katz M. // Geom. Funct. Anal. 1994. V. 4. P. 621-632. [6] Babenko I., Katz M. // Ann. Sci. Ecole Norm. Sup. (4). 1998. V. 31. P. 787-809. [7] Babenko I., Katz M., Suciu A. // Math. Res. Lett. 1998. V. 5. P. 461-471. [8] Katz M., Suciu A. // Contemp. Math. 1999. V. 231. P. 113-136. [9] Katz M., Suciu A. Systolic freedom of loop space // http://www.cs.bin.ac.il/ ${ }^{2}$ katzmik/ publications.html. [10] Gromov M. Metric Structures for Riemannian Spaces. Boston: Birkhäuser, 1999

Московский государственный

Принято редколлегией университет им. М. В. Ломоносова 07.09.2000 